\author{
Şermin ÇAM ÇELIK \\ Department of Mathematics, Faculty of Engineering and Natural Sciences, \\ Istanbul Bilgi University, Istanbul, TURKEY
}

\begin{abstract}
In this note, we obtain asymptotic results on integer parts of $\alpha p$ that are free of $k$ th powers of primes, where $p$ is a prime number and $\alpha$ is a positive real number.
\end{abstract}

\title{
1. Introduction and Statement of Results
}

Let $\alpha$ and $\beta$ be real numbers such that $\alpha>0$. Let $\lfloor x\rfloor$ denote the largest integer not greater than $x$. Sequences of the form $\{\lfloor\alpha n+\beta\rfloor\}_{n=1}^{\infty}$ are called Beatty sequences. A Beatty sequence is said to be homogeneous if $\beta=0$. Beatty sequences have been attracting a lot of attention since they can be viewed as analogues of arithmetic progressions, therefore they show up in a broad context. The interested reader is referred to $1,2,4,6,8,6,11,14,16,19,24$.

Let $k \geqslant 2$ be an integer. An integer is said to be $k$-free if it is not divisible by a $k$ th power of a prime. Very recently in [3, an asymptotic formula with an explicit error term is obtained for $k$-free values of homogeneous Beatty sequences at prime arguments (i.e. sequences of the form $\{\lfloor\alpha p\rfloor\}_{p=2}^{\infty}$ ) provided that $\alpha$ is of finite type (see Definition 1). This result can be viewed as a natural analogue of the result of Mirsky 20. In this article, we pursue this result and obtain two asymptotic formulas that are of the same flavour. The results we present here are well applicable to non-homogeneous Beatty sequences.

Theorem 1. Let $k \geq 2$ be an integer. Let $\left\{\alpha_{i}\right\}_{i=1}^{\ell}$ be a finite type subset of irrational numbers each greater than one. Assume that $\left\{\alpha_{i}\right\}_{i=1}^{\ell}$ satisfies (1) for some $\tau>0$. Let $\boldsymbol{\alpha}=\left(\alpha_{1}, \alpha_{2}, \ldots, \alpha_{\ell}\right)$ and

$$
\pi(x, k, \boldsymbol{\alpha})=\#\left\{p \leqslant x:\left\lfloor\alpha_{i} p\right\rfloor \text { is } k \text {-free for each } i=1, \ldots, \ell\right\} .
$$

2020 Mathematics Subject Classification. Primary 11N25; Secondary 11L20, 11L07, 11N60.

Keywords. Beatty sequences, exponential sums over primes, $k$-free numbers.

๑sermin.celik@bilgi.edu.tr

(D) 0000-0002-0110-7139. 
Then the following asymptotic is satisfied:

$$
\pi(x, k, \boldsymbol{\alpha})=\frac{\pi(x)}{\zeta^{\ell}(k)}+O\left(x^{1-\frac{k-1}{(k-1+\ell)(3 \tau+2)+k(\ell-1) \tau+k \ell}} e^{\frac{C \log x}{\log \log x}}\right)
$$

for some constant $C=C\left(\alpha_{1}, \ldots, \alpha_{\ell}\right)$ and every large $x$.

A nested version of Theorem 1 is given below.

Theorem 2. Let $k \geqslant 2$ be an integer. Let $\left\{\alpha_{1} \alpha_{2}, \alpha_{2}\right\}$ be a finite type subset of irrational numbers each greater than zero. Then the following asymptotic is satisfied:

$$
\#\left\{p \leqslant x:\left\lfloor\alpha_{1}\left\lfloor\alpha_{2} p\right\rfloor\right\rfloor \text { is } k \text {-free }\right\}=\frac{\pi(x)}{\zeta(k)}+O\left(x^{1-\varepsilon}\right)
$$

for some $\varepsilon>0$.

Here, the interested reader is invited to investigate the following problem: Let $\left\{\alpha_{i}\right\}_{i=1}^{n}$ be positive real numbers. Define

$$
a_{j}=\prod_{i=1}^{j} \alpha_{n+1-i} .
$$

Assuming that $\left\{a_{1}, a_{2}, \ldots, a_{n}\right\}$ is of finite type (see Definition 1 , show that

$$
\#\left\{p \leqslant x:\left\lfloor a_{n}\left\lfloor a_{n-1} \cdots\left\lfloor a_{1} p\right\rfloor\right\rfloor\right\rfloor \text { is } k \text {-free }\right\}=\frac{\pi(x)}{\zeta(k)}+O\left(x^{1-\varepsilon}\right)
$$

for some $\varepsilon>0$. It might also be fruitful to investigate the possible power saving in the error term above.

\subsection{Preliminaries and Notation.}

1.1.1. Notation. We recall that for functions $F$ and $G$ where $G$ is real non-negative, the notations $F \ll G$ and $F=O(G)$ are equivalent to the statement that the inequality $|F| \leqslant \alpha G$ holds for some constant $\alpha>0$. Further we use $F \sim G$ to indicate $(F / G)(x)$ tends to 1 as $x \rightarrow \infty$.

Given a real number $x$, we use the notation $\{x\}$ for the fractional part of $x$, the notation $\lfloor x\rfloor$ for the greatest integer not exceeding $x$ and $e(x)=e^{2 \pi i x}$.

We use $\|x\|$ to denote the distance from the real number $x$ to the nearest integer. $\Lambda(n)=\log p$ if $n=p^{r}$ where $p$ is a prime number (here and hereafter). Otherwise, $\Lambda(n)=0 . \mu(n)$ denotes the Mobius function. $\phi(n)$ denotes the Euler's totient function. $\tau(n)$ denotes the number of positive divisors of $n$. We also use $\pi(x)$ to denote the number of primes not more than $x$. 


\subsubsection{Preliminaries.}

Definition 1. An irrational number $\alpha$ is called of finite type $\tau$, if

$$
\tau=\sup \left\{\beta: \liminf _{\substack{q \rightarrow \infty \\ q \in \mathbb{N}}} q^{\beta}\|\alpha q\|=0\right\}<\infty .
$$

If $\alpha$ is an irrational number of finite type $\tau$, then by Dirichlet's approximation theorem (Lemma 2.1 of 25$]$ ) one has $\tau \geqslant 1$. The celebrated theorems of Khinchin 17] and of Roth 21,22 state that $\tau=1$ for almost all (in the sense of the Lebesque measure) real numbers and for all irrational algebraic numbers respectively.

Definition 2. A finite subset of real numbers $\left\{\beta_{1}, \beta_{2}, \ldots, \beta_{\ell}\right\}$ is said to be of finite type if there is $\tau>0$ such that the inequality

$$
\left\|h_{1} \beta_{1}+h_{2} \beta_{2}+\cdots+h_{\ell} \beta_{\ell}\right\|<\left(\max \left\{1,\left|h_{1}\right|, \ldots,\left|h_{\ell}\right|\right\}\right)^{-\tau}
$$

has only finitely many solutions for $h_{i} \in \mathbb{Z}$.

If $\left\{\beta_{i}\right\}_{i=1}^{\ell}$ satisfies (1) for some $\tau>0$, then it follows from Dirichlet's theorem on rational approximations that $\tau \geqslant 1$. Furthermore, such a set is linearly independent over $\mathbb{Q}$.

Throughout this paper, we shall mostly use the weak form of the prime number theorem, that is

$$
\pi(x) \sim \frac{x}{\log x}
$$

Lemma 1. For every positive integer $n \geq 1$,

$$
\tau(n)<e^{\frac{C \log 5 n}{\log \log 5 n}}
$$

for some constant $C>0$.

Proof. Follows from 23, Theorem 2.11].

Lemma 2. If

$$
\left|\alpha-\frac{a}{q}\right| \leqslant \frac{1}{q^{2}}
$$

for some integers $a$ and $q$ such that $(a, q)=1$, then

$$
\sum_{p \leqslant x} e(\alpha p) \ll x \log ^{3} x\left(q^{-\frac{1}{2}}+x^{-\frac{1}{5}}+q^{\frac{1}{2}} x^{-\frac{1}{2}}\right) .
$$

Proof. This follows in a standard way using the main result of $[12, \S 25]$.

Lemma 3 (Erdős-Turán-Koksma Inequality). If $\left\{\boldsymbol{x}_{i}\right\}_{i=1}^{N}$ is a finite sequence in $\mathbb{R}^{\ell}$, then for any $J \subseteq[0,1)^{\ell}$ that is a Cartesian product of subintervals of $[0,1)$ and any $H \geqslant 1$, we have

$$
\#\left\{1 \leqslant i \leqslant N: \boldsymbol{x}_{i} \in J \bmod 1\right\}-|J| N \ll \frac{N}{H}+\sum_{0<\|\boldsymbol{h}\| \leqslant H} \frac{1}{r(\boldsymbol{h})}\left|\sum_{1 \leqslant i \leqslant N} e\left(\left\langle\boldsymbol{h}, \boldsymbol{x}_{i}\right\rangle\right)\right| .
$$


Here $|J|$ denotes the $\ell$-dimensional Lebesgue measure of $J,\langle\cdot, \cdot\rangle$ denotes the standard inner product in $\mathbb{R}^{\ell}$ and we set $\|\boldsymbol{h}\|=\max _{1 \leqslant i \leqslant \ell}\left\{\left|h_{i}\right|\right\}$ and

$$
r(\boldsymbol{h})=\prod_{i=1}^{\ell} \max \left\{\left|h_{i}\right|, 1\right\}
$$

for all $\boldsymbol{h}=\left(h_{1}, h_{2}, \ldots, h_{\ell}\right) \in \mathbb{Z}^{\ell}$. Moreover, the implied constant depends only on $\ell$.

Proof. For the proof see 18 .

The following lemma is a classical result due to Vinogradov [26, Lemma 12].

Lemma 4. Let $\alpha, \beta$ and $\Delta$ be real numbers such that

$$
0<\Delta<\frac{1}{2} \text { and } \Delta \leqslant \beta-\alpha \leqslant 1-\Delta .
$$

Then there exists a periodic function $\Psi(x)$, with period 1 , satisfying

(i) $\Psi(x)=1$ in the interval $\alpha+\frac{1}{2} \Delta \leqslant x \leqslant \beta-\frac{1}{2} \Delta$,

(ii) $\Psi(x)=0$ in the interval $\beta+\frac{1}{2} \Delta \leqslant x \leqslant 1+\alpha-\frac{1}{2} \Delta$,

(iii) $0 \leqslant \Psi(x) \leqslant 1$ in the remainder of the interval $\alpha-\frac{1}{2} \Delta \leqslant x \leqslant 1+\alpha-\frac{1}{2} \Delta$,

(iv) $\Psi(x)$ has a Fourier expansion of the form

$$
\Psi(x)=\sum_{h=-\infty}^{\infty} a_{h} e(h x),
$$

where

$$
\left|a_{h}\right| \leqslant c \cdot \min \left\{|h|^{-1},|h|^{-2} \Delta^{-1}\right\}
$$

for every $|h| \geqslant 1$ and some $c$ fixed. Furthermore, $a_{0}=\beta-\alpha$.

\section{Proof of The Main Results}

2.1. Proof of Theorem 1. Let $\boldsymbol{\alpha}=\left(\alpha_{1}, \alpha_{2}, \ldots, \alpha_{\ell}\right)$ and

$$
\pi(x, k, \boldsymbol{\alpha})=\#\left\{p \leqslant x:\left\lfloor\alpha_{i} p\right\rfloor \text { is } k \text {-free for each } i=1, \ldots, \ell\right\} .
$$

Let $\mathcal{I}_{k}$ denote the characteristic function of $k$-free integers. Since

$$
\mathcal{I}_{k}(n)=\sum_{d^{k} \mid n} \mu(d)
$$

we have 


$$
\begin{aligned}
& \pi(x, k, \boldsymbol{\alpha}) \\
& =\sum_{p \leqslant x} \mathcal{I}_{k}\left(\left\lfloor\alpha_{1} p\right\rfloor\right) \cdots \mathcal{I}_{k}\left(\left\lfloor\alpha_{\ell} p\right\rfloor\right) \\
& =\sum_{p \leqslant x}\left(\sum_{d_{1}^{k}\left\lfloor\left\lfloor\alpha_{1} p\right\rfloor\right.} \mu\left(d_{1}\right)\right) \cdots\left(\sum_{d_{\ell}^{k} \backslash\left\lfloor\alpha_{\ell} p\right\rfloor} \mu\left(d_{\ell}\right)\right) \\
& =\sum_{\substack{\left.p \leqslant x \\
\left(d_{1}, \ldots, d_{\ell}\right) \\
d_{i}^{k} \mid \alpha_{i} p\right\rfloor \\
i=1, \ldots, \ell}} \mu\left(d_{1}\right) \cdots \mu\left(d_{\ell}\right) \\
& =\sum_{\left(d_{1}, \ldots, d_{\ell}\right)} \mu\left(d_{1}\right) \cdots \mu\left(d_{\ell}\right) \sum_{\substack{p \leqslant x \\
d_{i}^{k}\left\lfloor\left\lfloor\alpha_{i} p\right\rfloor \\
i=1, \ldots, \ell\right.}} 1 \\
& =\sum_{\substack{\left(d_{1}, \ldots, d_{\ell}\right) \\
d_{i} \leqslant z \\
i=1, \ldots, \ell}} \mu\left(d_{1}\right) \cdots \mu\left(d_{\ell}\right) \sum_{\substack{p \leqslant x \\
d_{i}^{k} \mid\left\lfloor\alpha_{i} p\right\rfloor \\
i=1, \ldots, \ell}} 1+\sum_{\begin{array}{c}
\left(d_{1}, \ldots, d_{\ell}\right) \\
d_{i}>z \\
\text { for some } i=1, \ldots, \ell
\end{array}} \mu\left(d_{1}\right) \cdots \mu\left(d_{\ell}\right) \sum_{\substack{p \leqslant x \\
d_{i}^{k} \|\left\lfloor\alpha_{i} p\right\rfloor \\
i=1, \ldots, \ell}} 1,
\end{aligned}
$$

where $z \leqslant x^{1 / k}$ will be chosen later. It follows from Lemma 1 that for all $i=$ $1,2, \ldots, \ell$ there exists a positive constant $c_{i}=c_{i}\left(\alpha_{i}\right)$ depending on $\alpha_{i}$ such that

$$
\tau\left(\left\lfloor\alpha_{i} p\right\rfloor\right) \ll e^{\frac{c_{i} \log x}{\log \log x}}
$$

for every $p \leqslant x$. Then, for all $i=1,2, \ldots, \ell$ and $p \leq x$

$$
\tau\left(\left\lfloor\alpha_{i} p\right\rfloor\right) \ll e^{\frac{c \log x}{\log \log x}},
$$

where $c=\max \left\{c_{1}, \ldots, c_{\ell}\right\}$. Set $C=c(\ell-1)$. Then, by (4) and using partial summation in the last step, we get

$$
\begin{aligned}
& \sum_{\substack{\left(d_{1}, \ldots, d_{\ell}\right) \\
d_{i}>z \\
\text { on some } i=1, \ldots, \ell}} \mu\left(d_{1}\right) \cdots \mu\left(d_{\ell}\right) \sum_{\substack{p \leqslant x \\
d_{i \mid l}^{k}\left\lfloor\alpha_{i} p\right\rfloor \\
i=1, \ldots, \ell}} 1 \\
& <\sum_{\substack{\left(d_{1}, \ldots, d_{\ell}\right) \\
d_{1}>z}} \sum_{\substack{p \leqslant x \\
d_{i}^{k} \backslash\left\lfloor\alpha_{i} p\right\rfloor \\
i=1, \ldots, \ell}} 1+\cdots+\sum_{\substack{\left(d_{1}, \ldots, d_{\ell}\right) \\
d_{\ell}>z}} \sum_{\substack{p \leqslant x \\
d_{i}^{k} \mid\left\lfloor\alpha_{i} p\right\rfloor \\
i=1, \ldots, \ell}} 1 \\
& =\sum_{p \leqslant x}\left(\sum_{\substack{d_{1}^{k}\left\lfloor\alpha_{1} p\right\rfloor \\
d_{1}>z}} 1\right) \cdots\left(\sum_{d_{\ell}^{k}\left\lfloor\left\lfloor\alpha_{\ell} p\right\rfloor\right.} 1\right)+\cdots+\sum_{p \leqslant x}\left(\sum_{d_{1}^{k}\left\lfloor\left\lfloor\alpha_{1} p\right\rfloor\right.} 1\right) \cdots\left(\sum_{\substack{d_{\ell}^{k}\left\lfloor\alpha_{\ell} p\right\rfloor \\
d_{\ell}>z}} 1\right)
\end{aligned}
$$




$$
\begin{aligned}
& \leqslant \sum_{p \leqslant x}\left(\sum_{\substack{d_{1}^{k}\left\lfloor\left\lfloor\alpha_{1} p\right\rfloor \\
d_{1}>z\right.}} 1\right)\left(\prod_{i=2}^{\ell} \tau\left(\left\lfloor\alpha_{i} p\right\rfloor\right)\right)+\cdots+\sum_{p \leqslant x}\left(\sum_{\substack{d_{\ell}^{k}\left\lfloor\left\lfloor\alpha_{\ell} p\right\rfloor \\
d_{\ell}>z\right.}} 1\right)\left(\prod_{i=1}^{\ell-1} \tau\left(\left\lfloor\alpha_{i} p\right\rfloor\right)\right) \\
& \ll e^{\frac{C \log x}{\log \log x}}\left(\sum_{p \leqslant x} \sum_{\substack{d_{1}^{k} \mid\left\lfloor\alpha_{1} p\right\rfloor \\
d_{1}>z}} 1+\cdots+\sum_{p \leqslant x} \sum_{d_{\ell}^{k}\left\lfloor\left\lfloor\alpha_{\ell} p\right\rfloor\right.} 1\right) \\
& \ll e^{\frac{C \log x}{\log \log x}}\left(\sum_{d_{1}>z} \sum_{\substack{p \leqslant x \\
d_{1}^{k}\left\lfloor\left\lfloor\alpha_{1} p\right\rfloor\right.}} 1+\cdots+\sum_{d_{\ell}>z} \sum_{\substack{p \leqslant x \\
d_{\ell}^{k} \mid\left\lfloor\alpha_{\ell} p\right\rfloor}} 1\right) \\
& \leqslant e^{\frac{C \log x}{\log \log x}}\left(\sum_{d_{1}>z} \sum_{\substack{m \leqslant \alpha_{1} x \\
d_{1}^{k} \mid m}} 1+\cdots+\sum_{d_{\ell}>z} \sum_{\substack{m \leqslant \alpha_{\ell} x \\
d_{\ell}^{k} \mid m}} 1\right) \\
& \leqslant e^{\frac{C \log x}{\log \log x}}\left(\sum_{d_{1}>z} \frac{\alpha_{1} x}{d_{1}^{k}}+\cdots+\sum_{d_{\ell}>z} \frac{\alpha_{\ell} x}{d_{\ell}^{k}}\right) \ll \frac{e^{\frac{C \log x}{\log \log x} x}}{z^{k-1}} .
\end{aligned}
$$

Therefore,

$$
\pi(x, k, \boldsymbol{\alpha})=\sum_{\substack{\left(d_{1}, \ldots, d_{\ell}\right) \\ d_{i} \leqslant z \\ i=1, \ldots, \ell}} \mu\left(d_{1}\right) \cdots \mu\left(d_{\ell}\right) \sum_{\substack{p \leqslant x \\ d_{i}^{k} \mid\left\lfloor\alpha_{i} p\right\rfloor \\ i=1, \ldots, \ell}} 1+O\left(\frac{e^{\frac{C \log x}{\log \log x} x}}{z^{k-1}}\right) .
$$

Next, we will study the sum above appearing in $(5)$ which runs over all tuples $\left(d_{1}, \ldots, d_{\ell}\right)$ of positive integers where $d_{i} \leqslant z$ for all $i=1, \ldots, \ell$.

So, let $\mathbf{d}=\left(d_{1}, \ldots, d_{\ell}\right)$ be such a tuple and set

$$
D=\prod_{j=1}^{\ell} d_{j}^{k}, \quad D_{i}=\prod_{\substack{j=1 \\ j \neq i}}^{\ell} d_{j}^{k} \quad \text { and } \quad \mathcal{I}_{\mathbf{d}}=\left[0, \frac{1}{d_{1}^{k}}\right) \times \cdots \times\left[0, \frac{1}{d_{\ell}^{k}}\right)
$$

for all $i=1, \ldots, \ell$. For a positive integer $i$, let $p_{i}$ denote the $i$ th prime. Observing that

$$
\lfloor\alpha p\rfloor \equiv 0 \quad(\bmod d) \text { if and only if }\left\{\frac{\alpha p}{d}\right\}<\frac{1}{d},
$$


we have

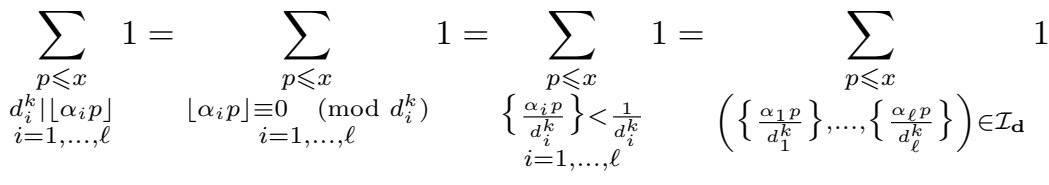

$$
\begin{aligned}
& =\#\left\{i \leqslant \pi(x): \mathbf{t}_{i} \in \mathcal{I}_{\mathbf{d}}\right\} \text {, }
\end{aligned}
$$

where

$$
\mathbf{t}_{i}=\left(\left\{\frac{\alpha_{1} p_{i}}{d_{1}^{k}}\right\}, \ldots,\left\{\frac{\alpha_{\ell} p_{i}}{d_{\ell}^{k}}\right\}\right) .
$$

It follows from Erdős-Turán-Koksma Inequality that for all $H \geqslant 1$,

$$
\begin{aligned}
& \#\left\{i \leqslant \pi(x): \mathbf{t}_{i} \in \mathcal{I}_{\mathbf{d}}\right\}-\frac{\pi(x)}{d_{1}^{k} \cdots d_{\ell}^{k}} \\
& \ll \frac{\pi(x)}{H}+\sum_{0<\|\mathbf{h}\| \leqslant H} \frac{1}{r(\mathbf{h})}\left|\sum_{i \leqslant \pi(x)} e\left(\left\langle\mathbf{h}, \mathbf{t}_{i}\right\rangle\right)\right| \\
& \ll \frac{\pi(x)}{H}+\sum_{0<\|\mathbf{h}\| \leqslant H} \frac{1}{r(\mathbf{h})}\left|\sum_{p \leqslant x} e\left(\frac{h_{1} D_{1} \alpha_{1}+\cdots+h_{\ell} D_{\ell} \alpha_{\ell}}{D} \cdot p\right)\right| .
\end{aligned}
$$

Next, we shall prove the following lemma.

\section{Lemma 5.}

$$
\begin{aligned}
& \sum_{p \leqslant x} e\left(\frac{h_{1} D_{1} \alpha_{1}+\cdots+h_{\ell} D_{\ell} \alpha_{\ell}}{D} \cdot p\right) \\
& \quad \ll x \log ^{3} x\left(x^{-\frac{1}{2(\tau+1)}}\left(\max \left\{\left|h_{1}\right| D_{1}, \ldots,\left|h_{\ell}\right| D_{\ell}\right\}\right)^{\frac{\tau}{2(\tau+1)}} D^{\frac{1}{2(\tau+1)}}+x^{-\frac{1}{5}}\right)
\end{aligned}
$$

uniformly for all $\mathbf{h}=\left(h_{1}, \ldots, h_{\ell}\right) \in \mathbb{Z}^{\ell}$ such that $\|\mathbf{h}\|>0$, where $D_{i}$ and $D$ are defined in (6).

Proof. Since $\left\{\alpha_{i}\right\}_{i=1}^{\ell}$ satisfies (1) for some $\tau>0$, there exists a positive constant $A \geq 1$ such that

$$
\left(\max \left\{\left|h_{1}\right|, \ldots,\left|h_{\ell}\right|\right\}\right)^{-\tau} \leqslant A\left\|h_{1} \alpha_{1}+h_{2} \alpha_{2}+\cdots+h_{\ell} \alpha_{\ell}\right\|
$$

for all $\left(h_{1}, \ldots, h_{\ell}\right) \in \mathbb{Z}^{\ell}$ such that $\max _{1 \leqslant i \leqslant \ell}\left\{\left|h_{i}\right|\right\}>0$. Let $\mathbf{h}=\left(h_{1}, \ldots, h_{\ell}\right) \in \mathbb{Z}^{\ell}$ be such a tuple and set

$$
m_{\mathbf{h}}=\frac{h_{1} D_{1} \alpha_{1}+\cdots+h_{\ell} D_{\ell} \alpha_{\ell}}{D} .
$$

Let $1 \leqslant Q<x / 2$ to be determined later. By Dirichlet's rational approximation theorem, there exists $\frac{r}{q} \in \mathbb{Q}$ such that $1 \leqslant q \leqslant \frac{x}{Q}$ and

$$
\left|m_{\mathbf{h}}-\frac{r}{q}\right|<\frac{Q}{q x} \text {. }
$$


So,

$$
\left\|q\left(h_{1} D_{1} \alpha_{1}+\cdots+h_{\ell} D_{\ell} \alpha_{\ell}\right)\right\|<\frac{Q D}{x} .
$$

On the other hand, it follows from $10 p$ that

$$
\left\|q\left(h_{1} D_{1} \alpha_{1}+\cdots+h_{\ell} D_{\ell} \alpha_{\ell}\right)\right\| \geqslant A^{-1} q^{-\tau}\left(\max \left\{\left|h_{1} D_{1}\right|, \ldots,\left|h_{\ell} D_{\ell}\right|\right\}\right)^{-\tau} .
$$

Combining (11) and (12), we get

$$
q \geqslant \frac{x^{\frac{1}{\tau}}}{\max \left\{\left|h_{1} D_{1}\right|, \ldots,\left|h_{\ell} D_{\ell}\right|\right\} A^{\frac{1}{\tau}} D^{\frac{1}{\tau}} Q^{\frac{1}{\tau}}} .
$$

Then it follows from Lemma 2 that

$$
\sum_{p \leqslant x} e\left(m_{\mathbf{h}} \cdot p\right) \ll x \log ^{3} x\left(x^{-\frac{1}{2 \tau}} M^{\frac{1}{2}} D^{\frac{1}{2 \tau}} Q^{\frac{1}{2 \tau}}+x^{-\frac{1}{5}}+Q^{-\frac{1}{2}}\right),
$$

where for the sake of brevity we set $M=\max \left\{\left|h_{1} D_{1}\right|, \ldots,\left|h_{\ell} D_{\ell}\right|\right\}$. By [13, Lemma $2.4]$, there exists $1 \leqslant Q<x / 2$ such that the left hand side of 114 is

$$
\ll x \log ^{3} x\left(x^{-\frac{1}{2(\tau+1)}} M^{\frac{\tau}{2(\tau+1)}} D^{\frac{1}{2(\tau+1)}}+x^{-\frac{1}{2 \tau}} M^{\frac{1}{2}} D^{\frac{1}{2 \tau}}+x^{-\frac{1}{5}}\right) .
$$

At this point, we can assume that $x^{-\frac{1}{2 \tau}} M^{\frac{1}{2}} D^{\frac{1}{2 \tau}}<1$, because otherwise the required upper bound holds trivially. Therefore, the second term is beaten by the first term giving the proof of Lemma 5 .

We next proceed by plugging this upper bound into (9). We also use the upper bound $\left|h_{i}\right| \leqslant H$ together with the upper bounds $D \leqslant z^{k \ell}$ and $D_{i} \leqslant z^{k(\ell-1)}$. Then the difference in the first line of $(9)$ is

$$
\ll \frac{\pi(x)}{H}+\left(x^{1-\frac{1}{2(\tau+1)}} H^{\frac{\tau}{2(\tau+1)}} z^{\frac{k(\ell-1) \tau+k \ell}{2(\tau+1)}} \log ^{3} x+x^{\frac{4}{5}} \log ^{3} x\right)\left(\sum_{0<\|\mathbf{h}\| \leqslant H} \frac{1}{r(\mathbf{h})}\right) .
$$

Now, by $(2)$

$$
\sum_{0<\|\mathbf{h}\| \leqslant H} \frac{1}{r(\mathbf{h})} \leqslant \sum_{0 \leqslant\|\mathbf{h}\| \leqslant H} \frac{1}{\prod_{i=1}^{\ell}\left(\max \left\{\left|h_{i}\right|, 1\right\}\right)} \leqslant\left(1+2 \sum_{1 \leqslant h \leqslant H} \frac{1}{h}\right)^{\ell} \ll \log ^{\ell} H
$$

where in the last step we use integral test. Here we note that the implied constant depends on $\ell$. Coupling (8), (9), (15) and (16), we arrive at

$$
\left(\sum_{\substack{p \leqslant x \\ d_{i}^{k}\left\lfloor\alpha_{i} p\right\rfloor \\ i=1, \ldots, \ell}} 1\right)-\frac{\pi(x)}{d_{1}^{k} \cdots d_{\ell}^{k}}
$$




$$
\ll \frac{\pi(x)}{H}+x^{1-\frac{1}{2(\tau+1)}} H^{\frac{\tau}{2(\tau+1)}} z^{\frac{k(\ell-1) \tau+k \ell}{2(\tau+1)}} \log ^{\ell} H \log ^{3} x+x^{\frac{4}{5}} \log ^{\ell} H \log ^{3} x
$$

for every $H \geqslant 1$ and every $\left(d_{1}, \ldots, d_{\ell}\right)$ such that $d_{i} \leqslant z \leqslant x^{1 / k}$ for each $i$. Noting $\pi(x) \ll x$ and choosing $1 \leqslant H \leqslant x$ by [13, Lemma 2.4], the left hand side of (17) is

$$
\ll \log ^{\ell+3} x\left(x^{1-\frac{1}{3 \tau+2}} z^{\frac{k(\ell-1) \tau+k \ell}{3 \tau+2}}+x^{1-\frac{1}{2(\tau+1)}} z^{\frac{k(\ell-1) \tau+k \ell}{2(\tau+1)}}+x^{\frac{4}{5}}\right) .
$$

On summing this over all tuples $\left(d_{1}, \ldots, d_{\ell}\right)$ of positive integers where $d_{i} \leqslant z$ for all $i=1, \ldots, \ell$, we observe from 5 that for all $1 \leqslant z \leqslant x^{1 / k}$,

$$
\begin{aligned}
& \pi(x, k, \boldsymbol{\alpha})-\pi(x) \sum_{\substack{\left(d_{1}, \ldots, d_{\ell}\right) \\
d_{i} \leqslant z \\
i=1, \ldots, \ell}} \frac{\mu\left(d_{1}\right) \cdots \mu\left(d_{\ell}\right)}{d_{1}^{k} \cdots d_{\ell}^{k}} \\
& \ll \log ^{\ell+3} x\left(x^{1-\frac{1}{3 \tau+2}} z^{\frac{k(\ell-1) \tau+k \ell}{3 \tau+2}+\ell}+x^{1-\frac{1}{2(\tau+1)}} z^{\frac{k(\ell-1) \tau+k \ell}{2(\tau+1)}+\ell}+x^{\frac{4}{5}} z^{\ell}\right)+\frac{e^{\frac{C \log x}{\log \log x}} x}{z^{k-1}} .
\end{aligned}
$$

Here,

$$
\sum_{\substack{\left(d_{1}, \ldots, d_{\ell}\right) \\ d_{i} \leqslant z \\ i=1, \ldots, \ell}} \frac{\mu\left(d_{1}\right) \cdots \mu\left(d_{\ell}\right)}{d_{1}^{k} \cdots d_{\ell}^{k}}=\left(\sum_{d \leqslant z} \frac{\mu(d)}{d^{k}}\right)^{\ell}
$$

and using the following inequality

$$
\left|\sum_{d \leqslant z} \frac{\mu(d)}{d^{k}}-\sum_{d=1}^{\infty} \frac{\mu(d)}{d^{k}}\right| \leqslant \sum_{d>z} \frac{1}{d^{k}} \ll \frac{1}{z^{k-1}},
$$

it follows by the mean value theorem that

$$
\left(\sum_{d \leqslant z} \frac{\mu(d)}{d^{k}}\right)^{\ell}-\left(\sum_{d=1}^{\infty} \frac{\mu(d)}{d^{k}}\right)^{\ell} \ll \frac{1}{z^{k-1}} .
$$

Therefore, the contribution of the sums running over $d_{i} \leqslant z$ for all $i=1, \ldots, \ell$ is

$$
\frac{\pi(x)}{\zeta^{\ell}(k)}+O\left(\frac{\pi(x)}{z^{k-1}}\right)
$$

yielding for all $1 \leqslant z \leqslant x^{1 / k}$

$$
\begin{aligned}
& \pi(x, k, \boldsymbol{\alpha})-\frac{\pi(x)}{\zeta^{\ell}(k)} \\
& \ll \log ^{\ell+3} x\left(x^{1-\frac{1}{3 \tau+2}} z^{\frac{k(\ell-1) \tau+k \ell}{3 \tau+2}+\ell}+x^{1-\frac{1}{2(\tau+1)}} z^{\frac{k(\ell-1) \tau+k \ell}{2(\tau+1)}+\ell}+x^{\frac{4}{5}} z^{\ell}\right)+\frac{e^{\frac{C \log x}{\log \log x}} x}{z^{k-1}},
\end{aligned}
$$


where $C=C(\ell, \boldsymbol{\alpha})$ is positive. On the right hand side of $(18)$, the first term beats the third term as $\tau \geq 1$ and the second term whenever

$$
z \leqslant x^{\frac{1}{\overline{k(\ell-1) \tau+k \ell}}}
$$

which one can assume since otherwise 18 holds trivially. Using now 13 Lemma 2.4 ] to choose optimal $z \leqslant x^{1 / k}$, the left hand side of $(18)$ is

$$
\begin{aligned}
& \ll e^{\frac{C^{\prime} \log x}{\log \log x}}\left(x^{1-\frac{1}{3 \tau+2}}+x^{\frac{1}{k}}+x^{\frac{(k-1)(3 \tau+1)+k(\ell-1) \tau+k \ell+\ell(3 \tau+2)}{(k-1)(3 \tau+2)+k(\ell-1) \tau+k \ell+\ell(3 \tau+2)}}\right) \\
& \ll x^{1-\frac{k-1}{(k-1+\ell)(3 \tau+2)+k(\ell-1) \tau+k \ell}} e^{\frac{C^{\prime} \log x}{\log \log x}}
\end{aligned}
$$

for some constant $C^{\prime}$ depending on $\ell$ and $\boldsymbol{\alpha}$, therefore the claim follows.

2.2. Proof of Theorem 2. The proof will be similar to that of Theorem 11. We shall therefore be brief. Let $\boldsymbol{\alpha}=\left(\alpha_{1}, \alpha_{2}\right)$ and define

$$
\pi_{\boldsymbol{\alpha}}(x, k)=\#\left\{p \leqslant x:\left\lfloor\alpha_{1}\left\lfloor\alpha_{2} p\right\rfloor\right\rfloor \text { is } k \text {-free }\right\} .
$$

Let $1 \leqslant z \leqslant x^{1 / k}$ be a number to be determined. Using (3), it follows that

$$
\pi_{\boldsymbol{\alpha}}(x, k)=\sum_{p \leqslant x} \sum_{d^{k} \mid\left\lfloor\alpha_{1}\left\lfloor\alpha_{2} p\right\rfloor\right\rfloor} \mu(d)=\sum_{p \leqslant x} \sum_{d^{k} \mid\left\lfloor\alpha_{1}\left\lfloor\alpha_{2} p\right\rfloor\right\rfloor} \mu(d)+\sum_{p \leqslant z} \sum_{\substack{d \leqslant x \\ d^{k} \backslash\left\lfloor\alpha_{1}\left\lfloor\alpha_{2} p\right\rfloor\right\rfloor \\ d>z}} \mu(d) .
$$

As we did before, we have

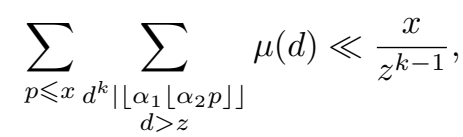

where the implied constant depends only on $\alpha_{1}$ and $\alpha_{2}$. This yields

$$
\pi_{\boldsymbol{\alpha}}(x, k)=\sum_{p \leqslant x} \sum_{\substack{d^{k}\left\lfloor\alpha_{1}\left\lfloor\alpha_{2} p\right\rfloor\right\rfloor \\ d \leqslant z}} \mu(d)+O\left(\frac{x}{z^{k-1}}\right) .
$$

We now proceed to derive the main term. Writing

$$
\left.\sum_{p \leqslant x} \sum_{\substack{d^{k}\left\lfloor\alpha_{1}\left\lfloor\alpha_{2} p\right\rfloor\right\rfloor \\ d \leqslant z}} \mu(d)=\sum_{d \leqslant z} \mu(d)\left(\sum_{\substack{p \leqslant x \\\left\lfloor\alpha_{1}\left\lfloor\alpha_{2} p\right\rfloor \equiv 0\right.}} 1\right)-\frac{\pi(x)}{d^{k}}\right)+\pi(x) \sum_{d \leqslant z} \frac{\mu(d)}{d^{k}},
$$

and using partial summation to get

$$
\sum_{d \leqslant z} \frac{\mu(d)}{d^{k}}=\frac{1}{\zeta(k)}+O\left(\frac{1}{z^{k-1}}\right)
$$


one arrives at

$$
\pi_{\boldsymbol{\alpha}}(x, k)=\frac{\pi(x)}{\zeta(k)}+O\left(\frac{x}{z^{k-1}}+\sum_{d \leqslant z}\left|\left(\sum_{\substack{p \leqslant x \\\left\lfloor\alpha_{1}\left\lfloor\alpha_{2} p\right\rfloor\right\rfloor \equiv 0\left(\bmod d^{k}\right)}} 1\right)-\frac{\pi(x)}{d^{k}}\right|\right)
$$

for any $1 \leqslant z \leqslant x^{1 / k}$. Let us now concentrate on the error term and proceed to show that it is $\ll x^{1-\varepsilon}$ for some $\varepsilon>0$. Using observation (7), together with Lemma 3 one ends up with

$$
\left(\sum_{\substack{p \leqslant x \\\left\lfloor\alpha_{1}\left\lfloor\alpha_{2} p\right\rfloor\right\rfloor \equiv 0}} 1\right)-\frac{\pi(x)}{d^{k}} \ll \frac{\pi(x)}{H_{1}}+\sum_{1 \leqslant\left|h_{1}\right| \leqslant H_{1}} \frac{1}{\left|h_{1}\right|}\left|\sum_{p \leqslant x} e\left(\frac{\alpha_{1} h_{1}\left\lfloor\alpha_{2} p\right\rfloor}{d^{k}}\right)\right|,
$$

where $H_{1}$ is a positive number to be determined. So, it boils down to estimate the exponential sum above. To do this, we let $K$ be a sufficiently large number and we write

$$
\left\lfloor\alpha_{2} p\right\rfloor=\alpha_{2} p-\left\{\alpha_{2} p\right\}
$$

yielding

$$
\sum_{p \leqslant x} e\left(\frac{\alpha_{1} h_{1}\left\lfloor\alpha_{2} p\right\rfloor}{d^{k}}\right)=\sum_{0 \leqslant i \leqslant K-1} \sum_{p \in I_{i}(x)} e\left(\frac{\alpha_{1} \alpha_{2} h_{1} p}{d^{k}}-\frac{\alpha_{1} h_{1}\left\{\alpha_{2} p\right\}}{d^{k}}\right),
$$

where $I_{i}(x)=\left\{p \leqslant x: \frac{i}{K} \leqslant\left\{\alpha_{2} p\right\}<\frac{i+1}{K}\right\}$. Since

$$
e(t)=1+O(|t|)
$$

uniformly for all $t \in \mathbb{R}$, we have

$$
e\left(\frac{\alpha_{1} \alpha_{2} h_{1} p}{d^{k}}-\frac{\alpha_{1} h_{1}\left\{\alpha_{2} p\right\}}{d^{k}}\right)=e\left(-\frac{\alpha_{1} h_{1} i}{K d^{k}}\right)\left(e\left(\frac{\alpha_{1} \alpha_{2} h_{1} p}{d^{k}}\right)+O\left(\frac{\left|h_{1}\right|}{K d^{k}}\right)\right)
$$

if $p \in I_{i}(x)$. Therefore, the left hand side of (21) is

$$
\ll \frac{\left|h_{1}\right| \pi(x)}{K d^{k}}+\sum_{0 \leqslant i \leqslant K-1}\left|\sum_{p \in I_{i}(x)} e\left(\frac{\alpha_{1} \alpha_{2} h_{1} p}{d^{k}}\right)\right| .
$$

Given $0 \leqslant i \leqslant K-1$, let $\beta_{i}=i / K, \gamma_{i}=(i+1) / K$ and $0<\Delta<1 / K$ be a number to be chosen. By Lemma there exists a periodic function $\Psi_{i}(x)$, with period 1 , satisfying

(i) $\Psi_{i}(x)=1$ in the interval $\beta_{i}+\frac{1}{2} \Delta \leqslant x \leqslant \gamma_{i}-\frac{1}{2} \Delta$,

(ii) $\Psi_{i}(x)=0$ in the interval $\gamma_{i}+\frac{1}{2} \Delta \leqslant x \leqslant 1+\beta_{i}-\frac{1}{2} \Delta$, 
(iii) $0 \leqslant \Psi_{i}(x) \leqslant 1$ in the remainder of the interval $\beta_{i}-\frac{1}{2} \Delta \leqslant x \leqslant 1+\beta_{i}-\frac{1}{2} \Delta$,

(iv) $\Psi_{i}(x)$ has a Fourier expansion of the form

$$
\Psi_{i}(x)=\sum_{h=-\infty}^{\infty} a_{h} e(h x),
$$

where $a_{0}=1 / K$ and

$$
\left|a_{h}\right| \leqslant c \cdot \min \left\{|h|^{-1},|h|^{-2} \Delta^{-1}\right\}
$$

for every $|h| \geqslant 1$ and some $c$ fixed.

Let $\psi_{i}(x)$ be 1 if $\beta_{i} \leqslant\{x\} \leqslant \gamma_{i}$ and $\psi_{i}(x)=0$ otherwise. It follows that $\Psi_{i}(x)$ and $\psi_{i}(x)$ agree on $[0,1]$ except possibly for two subintervals of $[0,1]$ of length $\leqslant \Delta$. Therefore,

$$
\sum_{p \in I_{i}(x)} e\left(\frac{\alpha_{1} \alpha_{2} h_{1} p}{d^{k}}\right)=\sum_{p \leqslant x} \Psi_{i}\left(\alpha_{2} p\right) e\left(\frac{\alpha_{1} \alpha_{2} h_{1} p}{d^{k}}\right)+O\left(\sum_{\substack{p \leqslant x \\\left\{\alpha_{2} p\right\} \in I}} 1\right)
$$

where $I$ is a union of two intervals and is of length $\Delta$. Since $\alpha_{2}$ is of finite type, following the proof of Theorem 5.1 in 8 together with a partial summation argument, it follows that for some $0<\varepsilon^{\prime \prime}<1 / 5$, one has

$$
\sum_{\substack{p \leqslant x \\\left\{\alpha_{2} p\right\} \in I}} 1=\Delta \pi(x)+O\left(x^{1-\varepsilon^{\prime \prime}}\right),
$$

uniformly for all $0<\Delta<1 / K$. Therefore, we see that the left hand side of (23) is

$$
\begin{aligned}
& =\frac{1}{K} \sum_{p \leqslant x} e\left(\frac{\alpha_{1} \alpha_{2} h_{1} p}{d^{k}}\right) \\
& \quad+O\left(\sum_{\left|h_{2}\right|>0}\left|a_{h_{2}}\right|\left|\sum_{p \leqslant x} e\left(\frac{\left(\alpha_{1} \alpha_{2} h_{1}+\alpha_{2} h_{2} d^{k}\right) p}{d^{k}}\right)\right|+\Delta \pi(x)+x^{1-\varepsilon^{\prime \prime}}\right) .
\end{aligned}
$$

Letting $\mathrm{H}_{2}$ be a positive integer to be determined, we split the sum running over $h_{2}$ at $H_{2}$. For $\left|h_{2}\right|>H_{2}$, estimating the innermost exponential sum by $\pi(x)$, and using the upper bounds $a_{h} \ll 1 /\left(\Delta h^{2}\right)$ and $a_{h} \ll 1 /|h|$, we obtain that the left hand side of 23 is

$$
\begin{array}{r}
=\frac{1}{K} \sum_{p \leqslant x} e\left(\frac{\alpha_{1} \alpha_{2} h_{1} p}{d^{k}}\right)+O\left(\sum_{0<\left|h_{2}\right| \leqslant H_{2}} \frac{1}{\left|h_{2}\right|}\left|\sum_{p \leqslant x} e\left(\frac{\left(\alpha_{1} \alpha_{2} h_{1}+\alpha_{2} h_{2} d^{k}\right) p}{d^{k}}\right)\right|\right) \\
+O\left(\frac{\pi(x)}{\Delta H_{2}}+\Delta \pi(x)+x^{1-\varepsilon^{\prime \prime}}\right) .
\end{array}
$$


Plugging this upper bound into 22 yields that

$$
\begin{aligned}
& \sum_{p \leqslant x} e\left(\frac{\alpha_{1} h_{1}\left\lfloor\alpha_{2} p\right\rfloor}{d^{k}}\right) \\
& \ll\left|\sum_{p \leqslant x} e\left(\frac{\alpha_{1} \alpha_{2} h_{1} p}{d^{k}}\right)\right|+\sum_{i \leqslant K} \sum_{0<\left|h_{2}\right| \leqslant H_{2}} \frac{1}{\left|h_{2}\right|}\left|\sum_{p \leqslant x} e\left(\frac{\left(\alpha_{1} \alpha_{2} h_{1}+\alpha_{2} h_{2} d^{k}\right) p}{d^{k}}\right)\right| \\
& +\frac{\pi(x) K}{\Delta H_{2}}+\Delta K \pi(x)+K x^{1-\varepsilon^{\prime \prime}}+\frac{\left|h_{1}\right| \pi(x)}{K d^{k}} .
\end{aligned}
$$

We are therefore left with the estimation of

$$
\sum_{p \leqslant x} e\left(\frac{\left(\alpha_{1} \alpha_{2} h_{1}+\alpha_{2} h_{2} d^{k}\right) p}{d^{k}}\right)
$$

whenever $\max \left\{\left|h_{1}\right|,\left|h_{2}\right|\right\}>0$. To estimate the exponential sum, by Dirichlet's theorem we pick up a rational number $a / q$ satisfying

$$
\left|\frac{\left(\alpha_{1} \alpha_{2} h_{1}+\alpha_{2} h_{2} d^{k}\right)}{d^{k}}-\frac{a}{q}\right|<\frac{1}{q x^{1-\kappa}}
$$

with $1 \leqslant q \leqslant x^{1-\kappa}$, where $0<\kappa<1$ is to be determined. Since $\left\{\alpha_{1} \alpha_{2}, \alpha_{2}\right\}$ is of finite type, similar to how we obtain (13)

$$
\frac{x^{\frac{1-\kappa}{\tau}}}{d^{\frac{k}{\tau}} \max \left\{\left|h_{1}\right|,\left|h_{2} d^{k}\right|\right\}} \ll q \leqslant x^{1-\kappa}
$$

for some $\tau \geqslant 1$. Then by Lemma 2 , the exponential sum 26 is

$$
\ll x \log ^{3} x\left(\left(\max \left\{\left|h_{1}\right|,\left|h_{2} d^{k}\right|\right\}\right)^{\frac{1}{2}} d^{\frac{k}{2 \tau}} x^{-\frac{1-\kappa}{2 \tau}}+x^{-\frac{1}{5}}+x^{-\frac{\kappa}{2}}\right) .
$$

At this point, we assume that $0<\max \left\{\left|h_{1}\right|,\left|h_{2}\right|\right\} \leqslant x^{\varepsilon^{\prime}}$ where $\varepsilon^{\prime}$ is a sufficiently small number to be determined in terms of $\kappa$. Then,

$$
\sum_{p \leqslant x} e\left(\frac{\left(\alpha_{1} \alpha_{2} h_{1}+\alpha_{2} h_{2} d^{k}\right) p}{d^{k}}\right) \ll\left(d^{\frac{k \tau+k}{2 \tau}} x^{1-\frac{1-\kappa}{2 \tau}+\frac{\varepsilon^{\prime}}{2}}+x^{\frac{4}{5}}+x^{1-\frac{\kappa}{2}}\right) \log ^{3} x,
$$

uniformly for

$$
0<\max \left\{\left|h_{1}\right|,\left|h_{2}\right|\right\} \leqslant x^{\varepsilon^{\prime}} .
$$

Plugging the upper bound 27) into 25], we arrive at

$$
\begin{aligned}
\sum_{p \leqslant x} e\left(\frac{\alpha_{1} h_{1}\left\lfloor\alpha_{2} p\right\rfloor}{d^{k}}\right) \ll K\left(d^{\frac{k \tau+k}{2 \tau}} x^{1-\frac{1-\kappa}{2 \tau}+\frac{\varepsilon^{\prime}}{2}}+x^{\frac{4}{5}}+x^{1-\frac{\kappa}{2}}\right) \log ^{4} x & \\
+ & \frac{\pi(x) K}{\Delta H_{2}}+\Delta K \pi(x)+K x^{1-\varepsilon^{\prime \prime}}+\frac{H_{1} \pi(x)}{K d^{k}},
\end{aligned}
$$


uniformly for $\left|h_{1}\right| \leqslant x^{\varepsilon^{\prime}}$, provided that $H_{2} \leqslant x^{\varepsilon^{\prime}}, 0<\kappa<1,0<\Delta<1 / K$ and $K$ is sufficiently large. Plugging this upper bound into 20 and summing over $d \leqslant z$, we see that the error term in 190 is

$$
\begin{aligned}
& \ll \frac{x z}{H_{1}}+K\left(z^{1+\frac{k \tau+k}{2 \tau}}\right.\left.x^{1-\frac{1-\kappa}{2 \tau}+\frac{\varepsilon^{\prime}}{2}}+z x^{\frac{4}{5}}+z x^{1-\frac{\kappa}{2}}\right) \log ^{5} x \\
&+\left(\frac{z x K}{\Delta H_{2}}+z \Delta K x+z K x^{1-\varepsilon^{\prime \prime}}+\frac{H_{1} x}{K}\right) \log x+\frac{x}{z^{k-1}}
\end{aligned}
$$

provided that $0<H_{1}, H_{2} \leqslant x^{\varepsilon^{\prime}}, 0<\kappa<1,0<\Delta<1 / K$ and $K$ is sufficiently large. We now make all unspecified constants explicit. For $0<\varepsilon_{1}, \varepsilon_{2}, \varepsilon_{3}, \varepsilon_{4}, \varepsilon_{5}<1$ to be determined, we set

$$
K=x^{\varepsilon_{1}}, H_{1}=x^{\varepsilon_{2}}, H_{2}=x^{\varepsilon_{3}}, \Delta=x^{-\varepsilon_{4}} \text { and } z=x^{\varepsilon_{5}},
$$

where $0<\varepsilon_{5} \leqslant 1 / k$ (this assumption is from the beginning of the proof). Examining each term in $(28)$, the right hand side of 28$]$ is $\ll x^{1-\varepsilon}$ for some $\varepsilon>0$, if the following inequalities are satisfied:

(1) $\varepsilon_{5}<1 / k$,

(2) $\varepsilon_{2}, \varepsilon_{3}<\varepsilon^{\prime}$

(3) $\varepsilon_{5}<\varepsilon_{2}<\varepsilon_{1}$,

(4) $\varepsilon_{1}+\varepsilon_{5}<\min \left\{\varepsilon_{4}, \varepsilon^{\prime \prime}, \kappa / 2\right\}$,

(5) $\varepsilon_{1}+\varepsilon_{4}+\varepsilon_{5}<\varepsilon_{3}$,

(6) $\varepsilon_{1}+\varepsilon_{5}\left(1+\frac{k \tau+k}{2 \tau}\right)+\frac{\varepsilon^{\prime}}{2}<\frac{1-\kappa}{2 \tau}$,

where $\varepsilon^{\prime \prime}<1 / 5$ is a fixed positive number defined in (24), $\tau \geqslant 1$ is a fixed number and $0<\kappa<1$ and $0<\varepsilon^{\prime}<1$ are to be chosen. We choose $\kappa=2 / 5$ and $\varepsilon^{\prime}=(1-\kappa) /(2 \tau)$. Then since $\varepsilon^{\prime \prime}<1 / 5$, we assume that $\varepsilon_{4}<\varepsilon^{\prime \prime}$ so that the fourth inequality becomes equivalent to $\varepsilon_{1}+\varepsilon_{5}<\varepsilon_{4}$. We next choose $\varepsilon_{3}<\varepsilon^{\prime}$ and $\varepsilon_{4}<\min \left\{\varepsilon_{3}, \varepsilon^{\prime \prime}\right\}$ and $\varepsilon_{1}<\min \left\{\varepsilon_{4}, \varepsilon_{3}-\varepsilon_{4},(1-\kappa) /(4 \tau)\right\}$. Finally, we choose $\varepsilon_{2}<\min \left\{\varepsilon_{1}, \varepsilon^{\prime}\right\}$ and

$$
\varepsilon_{5}<\min \left\{\varepsilon_{2}, \varepsilon_{4}-\varepsilon_{1}, \varepsilon_{3}-\varepsilon_{1}-\varepsilon_{4}, \frac{1}{k}, \frac{2 \tau}{(k+2) \tau+k}\left(\frac{1-\kappa}{4 \tau}-\varepsilon_{1}\right)\right\},
$$

completing the proof.

Declaration of Competing Interests The author declares that there are no conflicts of interest about the publication of this paper.

Acknowledgements The author thanks the referees for their comments which improved the quality of the paper. The author is supported by TÜBITAK Research Grant no. 119F425. 


\section{REFERENCES}

[1] Abercrombie, A. G., Beatty sequences and multiplicative number theory, Acta Arith., 70(3) (1995), 195-207. http://doi.org/10.4064/aa-70-3-195-207

[2] Abercrombie, A. G., Banks, W. D., Shparlinski, I. E., Arithmetic functions on Beatty sequences, Acta Arith. 136(1) (2009), 81-89. http://doi.org/10.4064/aa136-1-6

[3] Akbal, Y., A short note on some arithmetical properties of the integer part of $\alpha p$, Turkish Journal of Mathematics, 43(3) (2019), 1253-1262. http://doi.org/10.3906/mat-1809-43

[4] Akbal, Y., A note on values of Beatty sequences that are free of large prime factors, Colloquium Mathematicum, 160(1) (2020), 53-64. http://doi.org/10.4064/cm7715-2-2019

[5] Kumchev, A. V., On sums of primes from Beatty sequences, Integers 8 (2008), A8, 12 pp.

[6] Banks, W. D., Shparlinski, I. E., Non-residues and primitive roots in Beatty sequences, Bull. Austral. Math. Soc., 73(3) (2006), 433-443. http://doi.org/10.1017/S0004972700035449

[7] Banks, W. D., Shparlinski, I. E., Short character sums with Beatty sequences, Math. Res. Lett. 13 (2006), 539-547. http://doi.org/10.4310/MRL.2006.v13.n4.a4

[8] Banks, W. D., Shparlinski, I. E., Prime numbers with Beatty sequences, Colloq. Math. 115(2) (2009), 147-157. http://doi.org/10.4064/cm115-2-1

[9] Banks, W. D., Shparlinski, I. E., Prime divisors in Beatty sequences, J. Number Theory 123(2) (2007), 413-425. http://doi.org/10.1016/j.jnt.2006.07.011

[10] Banks, W. D., Güloğlu, A., Vaughan, R. C., Waring's problem for Beatty sequences and a local to global principle, J. Theor. Nombres Bordeaux 26(1) (2014), 1-16. http://doi.org/10.5802/jtnb.855

[11] Banks, W. D., Güloğlu, A., Nevans, C. W., Representations of integers as sums of primes from a Beatty sequence, Acta Arith., 130(3) (2007), 255-275. http://doi.org/10.4064/aa130-3-4

[12] Davenport, H., Multiplicative number theory, Third edition, Revised and with a preface by Hugh L. Montgomery, Graduate Texts in Mathematics, 74, Springer-Verlag, New York, 2000.

[13] Graham, S. W., Kolesnik, G., Van der Corput's method of exponential sums, London Mathematical Society Lecture Note Series, 126, Cambridge University Press, Cambridge, 1991.

[14] Güloğlu, A., Nevans, C. W., Sums of multiplicative functions over a Beatty sequence, Bull. Aust.Math. Soc. 78(2) (2008), 327-334. http://doi.org/10.1017/S0004972708000853

[15] Harman, G., Primes in Beatty sequences in short intervals, Mathematika, 62(2) (2016), 572586. http://doi.org/10.1112/S0025579315000376

[16] Harman, G., Primes in intersections of Beatty sequences, J. Integer Seq., 18(7) (2015), 12 p.

[17] Khinchin, A. Y., Zur metrischen Theorie der diophantischen Approximationen, Math. Z. 24(4) (1926), 706-714. http://doi.org/10.1007/BF01216806

[18] Koksma, J. F., Some Theorems on Diophantine Inequalities, Scriptum no. 5, Math. Centrmn Amsterdam, 1950

[19] Li, H., Pan, H., Primes of the form $\lfloor\alpha p+\beta\rfloor$, J. Number Theory, 129(10) (2009), 2328-2334.

[20] Mirsky, L., The number of representations of an integer as the sum of a prime and a $k$-free integer, Amer.Math. Monthly, 56 (1949), 17-19. http://doi.org/10.1080/00029890.1949.11990233

[21] Roth, K. F., Rational approximations to algebraic numbers, Mathematika, 2 (1955), 1-20. http://doi.org/10.1112/S0025579300000644

[22] Roth, K. F., Corrigendum to "Rational approximations to algebraic numbers", Mathematika, 2 (1955), 168.

[23] Montgomery, H. L., Vaughan, R. C., Multiplicative Number Theory I. Classical Theory, Cambridge University Press, Cambridge, 2007.

[24] Vaughan, R. C., The general Goldbach problem with Beatty primes, Ramanujan J., 34(3) (2014), 347-359. http://doi.org/10.1007/s11139-013-9501-3

[25] Vaughan, R. C., The Hardy-Littlewood Method, 2nd ed., Cambridge Univ. Press, 1997.

[26] Vinogradov, I. M. The Method of Trigonometrical Sums in the Theory of Numbers, Dover Publications, Inc., Mineola, NY, 2004. 